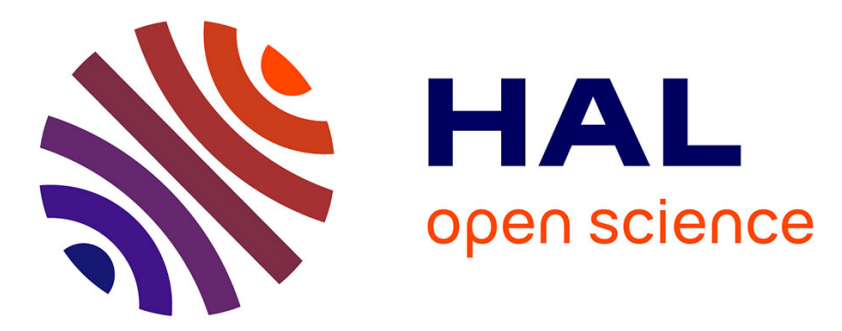

\title{
Impact of COVID-19 on the management of hepatocellular carcinoma in a high-prevalence area
}

Giuliana Amaddeo, Raffaele Brustia, Manon Allaire, Marie Lequoy, Clémence Hollande, Hélène Regnault, Lorraine Blaise, Nathalie Ganne-Carrié, Olivier Séror, Edouard Larrey, et al.

\section{To cite this version:}

Giuliana Amaddeo, Raffaele Brustia, Manon Allaire, Marie Lequoy, Clémence Hollande, et al.. Impact of COVID-19 on the management of hepatocellular carcinoma in a high-prevalence area. JHEP Reports Innovation in Hepatology, 2021, 3 (1), pp.100199. 10.1016/j.jhepr.2020.100199 . hal-03240158

\section{HAL Id: hal-03240158 \\ https://hal.science/hal-03240158}

Submitted on 28 May 2021

HAL is a multi-disciplinary open access archive for the deposit and dissemination of scientific research documents, whether they are published or not. The documents may come from teaching and research institutions in France or abroad, or from public or private research centers.
L'archive ouverte pluridisciplinaire HAL, est destinée au dépôt et à la diffusion de documents scientifiques de niveau recherche, publiés ou non, émanant des établissements d'enseignement et de recherche français ou étrangers, des laboratoires publics ou privés. 


\title{
Impact of COVID-19 on the management of hepatocellular carcinoma in a high-prevalence area
}

Giuliana Amaddeo, ${ }^{1,2,3, *, \dagger}$ Raffaele Brustia, ${ }^{4, \dagger}$ Manon Allaire, ${ }^{5,6}$ Marie Lequoy, ${ }^{7}$ Clémence Hollande, ${ }^{8}$ Hélène Regnault, ${ }^{1}$ Lorraine Blaise, ${ }^{9}$ Nathalie Ganne-Carrié, ${ }^{9,10}$ Olivier Séror ${ }^{10,11,12}$ Edouard Larrey, ${ }^{5,6}$ Chetana Lim, ${ }^{13}$ Olivier Scatton, ${ }^{6,13}$ Sanaa El Mouhadi, ${ }^{14}$ Violaine Ozenne, ${ }^{7}$ François Paye, ${ }^{15}$ Pierre Balladur, ${ }^{15}$ Anthony Dohan, ${ }^{16}$ Pierre-Philippe Massault, ${ }^{17}$ Stanislas Pol, ${ }^{8}$ Marco Dioguardi Burgio, ${ }^{11,12}$ Valérie Vilgrain, ${ }^{11,12}$ Ailton Sepulveda, ${ }^{18}$ Francois Cauchy, ${ }^{18}$ Alain Luciani, ${ }^{2,19}$ Daniele Sommacale, ${ }^{2,3,4}$ Vincent Leroy, ${ }^{1,2,3}$ Francoise Roudot-Thoraval, ${ }^{1}$ Mohamed Bouattour, ${ }^{20}$ Jean-Charles Nault ${ }^{9,10 * *}$, on behalf of the Paris Liver Cancer Group

\begin{abstract}
${ }^{1}$ Department of Hepatology, AP-HP, Hôpital Henri-Mondor, Créteil, France; ${ }^{2}$ Paris Est Créteil University, UPEC, Créteil, France; ${ }^{3}$ INSERM U955, Team "Pathophysiology and Therapy of Chronic Viral Hepatitis and Related Cancers", Assistance Publique-Hôpitaux de Paris, Créteil, France; ${ }^{4}$ Department of Digestive and Hepato-pancreatic-biliary Surgery, AP-HP, Hôpital Henri-Mondor, Créteil, France; ${ }^{5}$ Hepatology and Liver Transplantation Department, Hôpital Pitié Salpêtrière, Sorbonne Université, AP-HP, Paris, France; ${ }^{6}$ Sorbonne Université, Paris, France; ${ }^{7}$ Department of Hepatology, AP-HP, Hôpital Saint Antoine, Paris, France; ${ }^{8}$ Department of Hepatology, AP-HP, Hôpital Cochin, Paris, France; ${ }^{9}$ Service d'Hépatologie, Hôpital Jean Verdier, Hôpitaux Universitaires Paris-Seine-Saint-Denis, Assistance-Publique Hôpitaux de Paris, Bondy, France; ${ }^{10}$ Centre de Recherche des Cordeliers, INSERM, Sorbonne Université, Université Paris, INSERM UMR 1138 Functional Genomics of Solid Tumors Laboratory, Paris, France; ${ }^{11}$ Department of Radiology, Hôpital Beaujon, Hôpitaux Universitaires Paris Nord Val de Seine, Assistance-Publique Hôpitaux de Paris, Clichy, France; ${ }^{12}$ INSERM U1149 "Centre de Recherche sur l'Inflammation", CRI, Paris, France; ${ }^{13}$ Digestive and Liver Transplantation Department, Hôpital Pitié Salpêtrière, Sorbonne Université, AP-HP, Paris, France; ${ }^{14}$ Department of Radiology, AP-HP, Hôpital Saint Antoine, Paris, France; ${ }^{15}$ Department of Digestive Surgery, AP-HP, Hôpital Saint Antoine, Paris, France; ${ }^{16}$ Department of Radiology, AP-HP, Hôpital Cochin, Université de Paris, Paris, France; ${ }^{17}$ Department of Digestive Surgery, AP-HP, Hôpital Cochin, Université de Paris, Paris, France; ${ }^{18}$ Digestive and Liver Transplantation Department, Hôpital Beaujon, Hôpitaux Universitaires Paris Nord Val de Seine, Assistance-Publique Hôpitaux de Paris, Clichy, France; ${ }^{19}$ Department of Radiology, AP-HP, Hôpital Henri-Mondor, Créteil, France; ${ }^{20}$ Département d'Oncologie Digestive, AP-HP, Hôpital Beaujon, Clichy, France
\end{abstract}

\section{JHEP Reports 2021. https://doi.org/10.1016/j.jhepr.2020.100199}

Background \& Aims: Patients affected by hepatocellular carcinoma (HCC) represent a vulnerable population during the COVID-19 pandemic and may suffer from altered allocation of healthcare resources. The aim of this study was to determine the impact of the COVID-19 pandemic on the management of patients with HCC within 6 referral centres in the metropolitan area of Paris, France.

Methods: We performed a multicentre, retrospective, cross-sectional study on the management of patients with HCC during the first 6 weeks of the COVID-19 pandemic (exposed group), compared with the same period in 2019 (unexposed group). We included all patients discussed in multidisciplinary tumour board (MTB) meetings and/or patients undergoing a radiological or surgical programmed procedure during the study period, with curative or palliative intent. Endpoints were the number of patients with a modification in the treatment strategy, or a delay in decision-to-treat.

Results: After screening, $\mathrm{n}=670$ patients were included $(\mathrm{n}=293$ exposed to COVID, $\mathrm{n}=377$ unexposed to COVID). Fewer patients with HCC presented to the MTB in 2020 ( $p=0.034)$ and fewer had a first diagnosis of HCC ( $\mathrm{n}=104$ exposed to COVID, $\mathrm{n}=143$ unexposed to COVID, $p=0.083$ ). Treatment strategy was modified in $13.1 \%$ of patients, with no differences between the 2 periods. Nevertheless, $21.5 \%$ vs. 9.5\% of patients experienced a treatment delay longer than 1 month in 2020 compared with 2019 ( $p<0.001)$. In 2020, 7.1\% (21/293) of patients had a diagnosis of an active COVID-19 infection: $11(52.4 \%)$ patients were hospitalised and 4 (19.1\%) patients died.

Conclusions: In a metropolitan area highly impacted by the COVID-19 pandemic, we observed fewer patients with HCC, and similar rates of treatment modification, but with a significantly longer treatment delay in $2020 \mathrm{vs} .2019$.

(C) 2020 The Author(s). Published by Elsevier B.V. on behalf of European Association for the Study of the Liver (EASL). This is an open access article under the CC BY-NC-ND license (http://creativecommons.org/licenses/by-nc-nd/4.0/).

Keywords: 2019-nCoV; COVID-19; Management; Hepatocellular carcinoma; Cirrhosis.

Received 20 July 2020; received in revised form 29 September 2020; accepted 13 October 2020; available online 31 October 2020

+ These authors equally contributed to this study.

¥ Paris Liver Cancer Group: Olivier Sutter, Pierre Nahon, Marianne Ziol, Julien Calderaro, Alexis Laurent, Ariane Mallat, Christophe Duvoux, Vania Tacher, Vincent Mallet, Sebastien Gaujoux, Philippe Soyer, Benoit Terris, Olivier Soubrane, Maxime Ronot, Valérie Paradis, Lionel Arrivé, Dominique Wandum, Mathilde Wagner, Claire Goumard, Eric Savier.

* Corresponding authors. Addresses: Hepatology Department, Hôpitaux Universitaires Henri-Mondor, 51 avenue du Maréchal de Lattre de Tassigny, 94010 Créteil Cedex, France. Tel.: +33 649875673; fax: +3149812352. (G. Amaddeo), or Hepatology Department, Hôpital Jean Verdier, Avenue du 14 juillet, 93140 Bondy, France. Tel.: +33 6106794 61; fax: +3315372 51 92. (J.-C. Nault).

E-mail addresses: giuliana.amaddeo@aphp.fr (G. Amaddeo), naultjc@gmail.com (J.-C. Nault). 


\section{Introduction}

The COVID-19 pandemic, as declared by the World Health Organization, ${ }^{1}$ has no true precedent in modern times and is a rapidly evolving crisis worldwide. Cancer remains a heavy burden with more than 18 million cases diagnosed in 2018 according to the GLOBOCAN reports, with an estimated global prevalence beyond 43 million people. ${ }^{2}$

Cancer patients represent a vulnerable population because of their acquired immunodeficiency, and are at increased risk of COVID-19-related serious events (intensive care admission, requirement for mechanical ventilation, or death). ${ }^{3}$

In a report from China on 72,314 COVID-19-positive patients, the crude-fatality rate was $5.3 \%$ among cancer patients with higher mortality rates among those aged over 70 years. $^{4,5}$ In an Italian cohort of 335 infected patients, 20.3\% of patients with COVID-related disease who died had an active cancer. ${ }^{6}$

The context is complexified by the unusual altered allocation of healthcare resources to the pandemic, which might be responsible for collateral damage to the healthcare system on which patients depend: screening interruption, treatments cancelled or downgraded, follow up delay, and patient fear. ${ }^{7}$ According to the European Association for the Study of Liver (EASL) guidelines, management and care of patients affected by hepatocellular carcinoma (HCC), the fourth cause of cancer-related death worldwide, should be maintained, even with minimal exposure to medical staff, including systemic treatments and liver transplantation (LT) work up. ${ }^{8}$ Similarly, the French Association for the Study of the Liver board suggests to maintain the curative treatments of HCC in dedicated hospital units, separate from COVID-19 patients. ${ }^{9}$ In France, the metropolitan area of Paris $^{10}$ is heavily impacted by the outbreak and non-urgent procedures might be re-scheduled, with an approximate delay of 1-2 months. ${ }^{9}$

The aim of this study was to determine the impact of the COVID-19 pandemic on the management (compliance with multidisciplinary tumour board [MTB] decisions on the time and type of treatment) for patients affected by HCC within 6 French academic referral centres of the metropolitan area of Paris.

\section{Methods and analysis}

This study was designed as a multicentre, retrospective, crosssectional study on the management of patients affected by HCC during the first 6 weeks of the COVID-19 pandemic (from 6 March 2020 to 17 April 2020), compared with the same period in 2019 (from 6 March 2019 to 17 April 2019), within the metropolitan area of Paris.

Six academic referral centres from the AP-HP network (Pitié Salpêtrière-Paris, Saint-Antoine-Paris, Cochin-Paris, BeaujonClichy, Jean-Verdier-Bondy), including the steering committee (Hôpital Henri Mondor, Créteil) were involved in the study, organised during the first week of the French national first wave of the pandemic (6-14 March 2020).

Informed consent was obtained from all the participants; the study was approved by the Henri-Mondor Institutional Review Board (Ethics number committee 00011558, Approval Number 2020-071), and led in compliance with Strengthening the Reporting of Observational Studies in Epidemiology guidelines for cross-sectional studies. ${ }^{11}$

\section{Inclusion criteria}

- Adult patients ( $>18$ years old) affected by HCC (histological and/or radiological diagnosis according to the EASL criteria), ${ }^{12}$ who received during the inclusion period.

- A proposal of treatment in MTB meetings. OR

- A programmed surgical or radiological procedure, such as liver resection (LR) or any interventional radiology (IR) procedure: percutaneous ablation (radiofrequency, microwaves, or irreversible electroporation), trans-arterial-chemo-embolisation (TACE), or selective internal radiation therapy (SIRT).

\section{Study period}

Patients fulfilling the inclusion criteria and exposed to the pandemic between 6 March 2020 and 17 April 2020 were considered as cases (Exposed_COVID), and those fulfilling the same inclusion criteria between 6 March 2019 and 17 April 2019 (Unexposed_COVID) were considered as controls.

\section{Study endpoints}

The impact of the pandemic on the management of the target population affected by HCC was measured as the number of patients with a change in the treatment strategy (treatment realised was different from what was proposed in the MTB), during the 2 periods. We aimed to assess the incidence of COVID19 infection among the study cohort, MTB-to-treatment interval (days) between the MTB decision and treatment, the type of management (curative or palliative treatment) within the time periods for the exposed and control groups.

\section{Variables}

From the digital integrated care patient file (Orbis $\odot$, Agfa HealthCare, Mortsel, Belgium), we retrieved variables on general demographics, underlying liver disease, HCC characteristics (main tumour size, presence of tumour thrombosis, extrahepatic disease, and BCLC staging), serum alpha-fetoprotein level, and clinical management proposed within MTB meetings. The type of treatment proposed was detailed, as well as the treatment finally realised, and any potential delay between the date planned and the date treatment finally occurred. In cases where treatment did not occur, we estimated the decision-to-treatment delay from the MTB to the date of planned treatment. We also collected the reason for an alternative therapeutic decision and for a treatment delay. The reasons for outpatient cancellation were recorded, as well as the type of consultation (video/telemedicine or classical outpatient consultation).

Treatments proposals were classed as:

- Curative intent: surgery (LR, liver transplantation [LT]) or percutaneous ablation by IR (radiofrequency, microwaves, or irreversible electroporation);

- Palliative intent: systemic therapies including sorafenib, lenvantinib, immunotherapy, regorafenib, cabozantinib; external radiotherapy; TACE; SIRT;

- Best supportive care.

The above treatments were classed as interventional (surgery, IR, external radiotherapy, TACE, SIRT) or non-interventional (systemic therapies including sorafenib, levantinib, immunotherapy, regorafenib, cabozantinib). 


\section{JHEP|Reports}

The treatment strategy was considered 'downgraded' in case of treatment switch from 'curative intent' to 'palliative intent' or 'best supportive care'.

In the cohort of patients exposed to COVID-19 in 2020, were identified patients with a diagnosis of an active COVID-19 infection based on RT-PCR testing, suggestive chest CT, or typical COVID-19 symptoms (fever and upper respiratory tract symptoms - including anosmia, dysgeusia, fatigue, dry cough, and dyspnoea - with lymphopenia or leukopenia).

Data from each centre were entered in a single digital worksheet database, hosted on a secure computer. Dataset from each centre were harmonised and merged in a single dataset for analysis. Each patient was de-identified and assigned to an anonymised alpha-numeric code. The quality of data management was compliant to the reference methodology on personal data processing and protection (MR004), as stated by the French data protection authority (Commission Nationale de l'Informatique et des Libertés, CNIL n 2209983 v 0).

\section{Sample size}

No a priori sample size calculation was realised, and the whole cohort of patients according to the inclusion criteria was included.

\section{Statistical analysis}

Descriptive results were expressed as median with inter-quartile range (IQR) for continuous variables and as $\mathrm{n}(\%)$ for categorical data. Baseline characteristics of patients and HCC were compared between the 2 period cohorts using the Mann-Whitney $U$ test for continuous data, and $\chi^{2}$ test or Fisher exact test for qualitative variables. Variables significantly different between the 2 cohorts were used as variables for adjustment.

The main criteria and proportion of cases with modified treatment was compared between the 2 time period cohorts and expressed as crude odds ratio (OR) with 95\% CIs. Baseline characteristics found different between the 2 period cohorts (with $p$ $<0.10$ ) were tested in a logistic regression model giving adjusted OR (aOR) with 95\% CI. The main criteria were analysed in the whole study population proposed for surgical, radiological, and medical treatment and separately in patients with a first diagnosis of HCC and patients in follow up for a known HCC. A logistic regression model was used to test variables independently associated with main criteria and aORs are presented with $95 \%$ CI. No multiple imputations were used. A value of $p \leq 0.05$ was considered significant. All analyses were performed using IBM SPSS version 26 (IBM SPSS Inc., Chicago, IL, USA) and Stata version 13.0 (Statacorp, College Station, TX, USA).

\section{Results}

\section{General characteristics}

We screened 1661 patients in 6 centres for eligibility: either presented in a MTB meeting ( $\mathrm{n}=543$, Exposed_COVID; $\mathrm{n}=804$ Unexposed_COVID) or with a programmed surgical or radiological procedure with intention to treat between 6 March and 17 April in 2019 and 2020. A detailed flowchart is shown in Fig. 1. Of these patients, 723 were excluded because they were not affected by HCC, and a further 268 were excluded because of the absence of any treatment proposal (follow up or requiring further explorations). Six hundred and seventy patients were included, and represented the study population ( $n=293$ Exposed_COVID, $\mathrm{n}=377$ Unexposed_COVID). The percentage of males

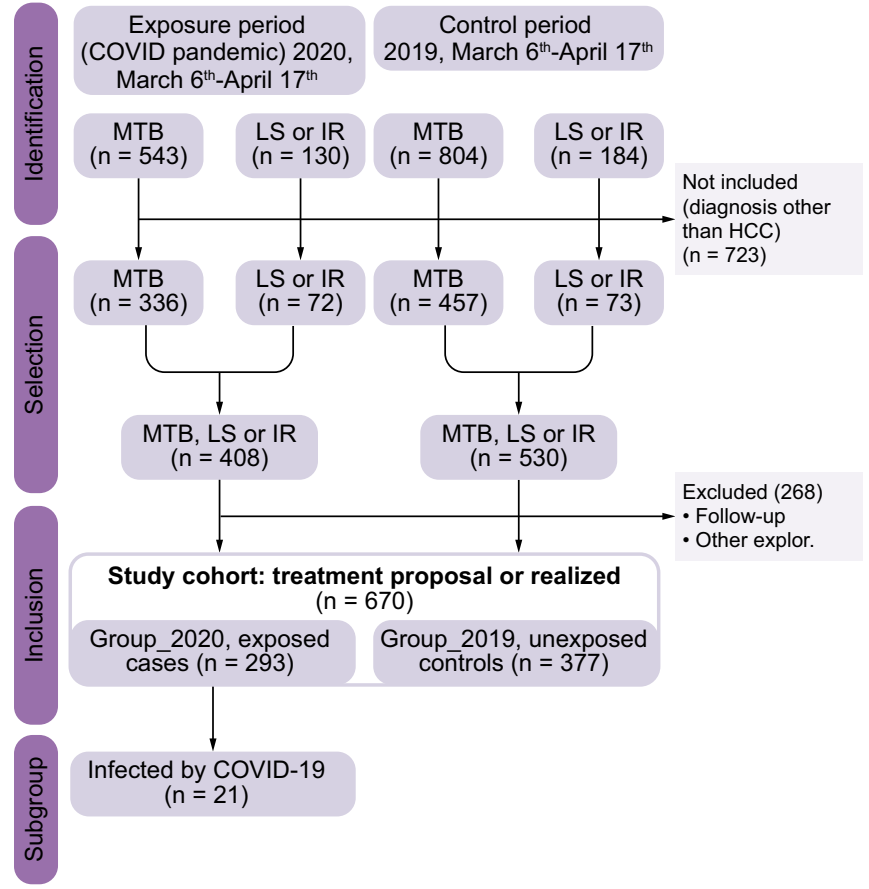

Fig. 1. Flow chart of patients included in the study. HCC, hepatocellular carcinoma; IR, interventional radiology; LS, liver surgery; MTB, multidisciplinary tumour board.

was $82.6 \%$, and the median age was 67 (60-74) years; no differences were observed in terms of patient characteristics (ChildPugh score, model for end-stage liver disease) or tumour patterns (maximum diameter, portal vein invasion, AFP) between the 2 periods (Table 1 ). Among the group of patients exposed to the pandemic, $7.1 \%(\mathrm{n}=21)$ had a diagnosis of COVID-19 infection.

\section{Impact of the pandemic on MTB meetings, treatments, and patient follow up}

The absolute number of patients affected by HCC - including those with a first diagnosis - presented to the MTB was lower in $2020(\mathrm{n}=221)$ compared with $2019(\mathrm{n}=304)$. This number significantly decreased over the weeks in 2020 but not in 2019 $(p=0.034)$, with a similar - but not significant - trend for those with a first diagnosis of HCC $(p=0.083)$ as detailed in Fig. 2 .

The rate of treatments (proposed or performed) in patients with active HCC during the inclusion period was $56.7 \%$ ( $\mathrm{n}=$ $377)$ in 2019 vs. $43.7 \%(n=293)$ in 2020 , with a significant decrease during the second half of the time period in 2020 ( $p=$ 0.018 ).

We observed no significant differences in the decision-totreatment interval between 2019 and 2020 (Table 1) for each treatment class. Nevertheless, a higher rate of patients experienced a treatment delay longer than 1 month in 2020 compared with 2019 (21.5\%, $\mathrm{n}=63$ vs. 9.5\%, $n=36$, respectively; $p<0.001$ ). The reasons for this delay were different according to the study period (Table 1). When focusing on the COVID-19 period, a higher rate of patients requiring an interventional procedure experienced a delay longer than 1 month (interventional procedure: $<1$ month, $n=100$ [54.3\%] vs. >1 month, $\mathrm{n}=75$ [68.8\%]), compared with those requiring medical treatment (medical 
Table 1. Baseline characteristics of 670 patients discussed by the MTB.

\begin{tabular}{|c|c|c|c|}
\hline & $\begin{array}{r}\text { Unexposed COVID-19 } \\
2019(n=377) \\
\end{array}$ & $\begin{array}{r}\text { Exposed COVID-19 } \\
2020(n=293)\end{array}$ & $p$ value $^{* *}$ \\
\hline Sex - male, n (\%) & $309(82.0)$ & $245(83.6)$ & 0.608 \\
\hline Age, years & $67(60-74)$ & $67(60-73)$ & 0.888 \\
\hline Cirrhosis, n (\%) & $317(84.1)$ & $243(82.9)$ & 0.753 \\
\hline \multicolumn{4}{|l|}{ Liver disease aetiology, n (\%) } \\
\hline $\mathrm{HCV}$ & $63(16.7)$ & $51(17.4)$ & \\
\hline HBV & $59(15.7)$ & $38(12.9)$ & \\
\hline $\mathrm{HCV}+\mathrm{HCV}$ & $41(10.9)$ & $44(15.0)$ & 0.569 \\
\hline Alcohol & $67(17.8)$ & $53(18.1)$ & \\
\hline NASH & $65(17.2)$ & $54(18.4)$ & \\
\hline Alcohol + NASH & $57(15.1)$ & $38(13.0)$ & \\
\hline Other & $25(6.6)$ & $15(5.1)$ & \\
\hline Tumour burden, $\mathrm{mm}(\mathrm{n}=638)$ & $30(18-57)$ & $30(18-56)$ & 0.582 \\
\hline \multicolumn{4}{|l|}{ BCLC classification } \\
\hline BCLC 0 & $35(9.3)$ & $28(9.6)$ & \\
\hline BCLC A & $133(35.2)$ & 109 (37.2) & \\
\hline BCLC B & $99(26.3)$ & $81(27.6)$ & 0.142 \\
\hline BCLC C & $84(22.3)$ & $51(17.4)$ & \\
\hline BCLC D & $26(6.9)$ & $24(8.2)$ & \\
\hline Tumour thrombosis $(n=667)$ & $58(15.5)$ & $43(14.7)$ & 0.828 \\
\hline \multicolumn{4}{|l|}{ Alpha-fetoprotein, ng/ml $(\mathrm{n}=653)$} \\
\hline$<10$ & $192(52.3)$ & $139(48.6)$ & 0.385 \\
\hline$>10$ & $175(47.7)$ & $147(51.4)$ & \\
\hline \multicolumn{4}{|l|}{ Inclusion criteria, n (\%) } \\
\hline LS and IR performed & $145(21.6)$ & $72(24.6)$ & 0.109 \\
\hline MTB discussion & $525(78.4)$ & $221(75.4)$ & \\
\hline \multicolumn{4}{|l|}{ Type of management, n (\%) } \\
\hline First diagnosis & $143(36.9)$ & $104(35.5)$ & 0.520 \\
\hline Follow up & $234(62.1)$ & $189(64.5)$ & \\
\hline \multicolumn{4}{|l|}{ Diagnostic modality, n (\%) } \\
\hline Imaging & $288(76.4)$ & $229(78.2)$ & 0.643 \\
\hline Histology & $89(23.6)$ & $64(21.8)$ & \\
\hline \multicolumn{4}{|l|}{ Imaging technique, $n(\%)(n=613)$} \\
\hline $\mathrm{CT}$ & $118(35.0)$ & $90(32.6)$ & \\
\hline MRI & $118(35.0)$ & $99(35.9)$ & 0.813 \\
\hline Both & $101(30.0)$ & $87(31.5)$ & \\
\hline \multicolumn{4}{|l|}{ Proposed treatment } \\
\hline Curative & $148(39.3)$ & $122(41.6)$ & \\
\hline Palliative & $185(49.1)$ & $138(47.1)$ & 0.830 \\
\hline BSC & $44(11.7)$ & $33(11.3)$ & \\
\hline \multicolumn{4}{|l|}{ Performed treatment } \\
\hline Curative & $137(36.3)$ & $101(34,5)$ & \\
\hline Palliative & $172(45.6)$ & $134(45.7)$ & 0.357 \\
\hline BSC & $65(17.2)$ & $36(12.3)$ & \\
\hline Planned but not realised & $3(0.8)$ & $21(5.3)$ & $<0.001$ \\
\hline Therapeutic protocol inclusion & $24(6.4)$ & $12(4.1)$ & 0.228 \\
\hline Treatment change & $49(13.0)$ & $39(13.3)$ & 0.909 \\
\hline \multicolumn{4}{|l|}{ Cause of treatment change } \\
\hline Disease progression & $32(65.3)$ & $9(23.1)$ & $<0.001$ \\
\hline Failed or contra-indicated treatment & $13(26.5)$ & $4(10.2)$ & \\
\hline Patient's choice & $4(8.1)$ & $8(20.5)$ & \\
\hline Related COVID-19 & 0 & $18(46.1)$ & \\
\hline \multicolumn{4}{|l|}{ MTB-to-treatment interval, days } \\
\hline Curative* & $32(23-55)$ & $31(18-53)$ & 0.324 \\
\hline Palliative & $25(10-36)$ & $26(13-38)$ & 0.786 \\
\hline BSC & $1(0-5)$ & $0(0-7)$ & 0.558 \\
\hline MTB-to-treatment interval $>1$ month & $36(9.5)$ & $63(21.5)$ & $<0.001$ \\
\hline Treatment related & $16 / 33(48.4)$ & $7 / 61(11.4)$ & \\
\hline Material related & $9(27.2)$ & $3 / 61(5)$ & $<0.001$ \\
\hline COVID-19 related & 0 & $47(77)$ & \\
\hline Patient related & $8(24.2)$ & $4 / 61(6.5)$ & \\
\hline \multicolumn{4}{|l|}{ Outpatient consultation } \\
\hline Cancelled & $5(1.4)$ & $21(7.8)$ & \\
\hline Standard & $364(97.3)$ & $165(56.5)$ & $<0.001$ \\
\hline Teleconsultation & $5(1.3)$ & $105(35.9)$ & \\
\hline
\end{tabular}

BSC, best supportive care; IR, interventional radiology; LS, liver surgery; LT, lung transplant; MTB, multidisciplinary tumour board, NASH, non-alcoholic steatohepatitis.

${ }^{* *}$ Categorical variables were compared using Fisher's exact test and continuous variables using Mann Whitney non parametric test.

* LT and the related work up were excluded. 


\section{JHEP|Reports}

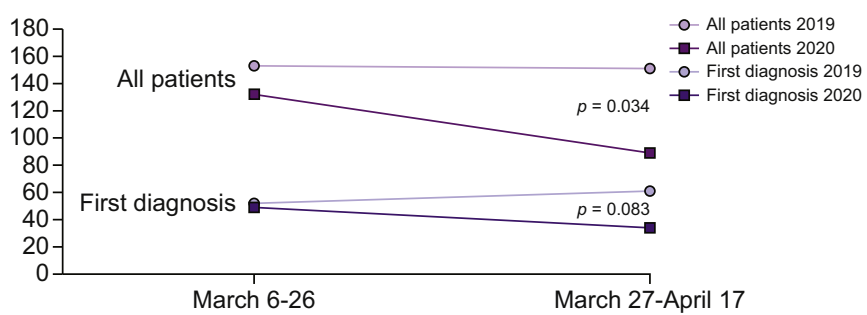

Fig. 2. Distribution of patients discussed in the MTB. Number of patients presented to the MTB over the same time periods during 2019 and 2020. Each period was split in half (first 3 weeks and second 3 weeks), and the distribution was compared. The numbers of patients with HCC presented to the MTB in 2020 decreased during the pandemic period: (for 2019: 6-26 March, $\mathrm{n}=$ 153; 27 March-7 April, $\mathrm{n}=151$; for 2020: 6-26 March, $\mathrm{n}=132 ; 27$ March-7 April, $\mathrm{n}=89$, Fisher's exact test, $p=0.034$ ). The number of patients presented to the MTB with a first diagnosis of HCC also decreased: (for 2019: 6-26 March, $\mathrm{n}=52$; 27 March-7 April, $\mathrm{n}=61$; for 2020: 6-26 March, $\mathrm{n}=49$, 27 March-7 April, $\mathrm{n}=34$, Fisher's exact test, $p=0.083$ ). MTB, multidisciplinary tumour board.

treatment: <1 month, $\mathrm{n}=77$ [41.8\%] vs. >1 month, $\mathrm{n}=15$ [13.8\%], Table S1).

Overall, 36 patients (5.4\%) accepted to be included within a study protocol, with no differences in the inclusion rates between the 2 periods $(\mathrm{n}=12,4.1 \% v s . \mathrm{n}=24,6.4 \%$ in $2020 v$ s. 2019 , respectively, $p=0.228$ ).

Finally, apart from a higher rate of cancelled consultations, the outpatient models have changed with a significantly greater use of teleconsultation during the pandemic $(7.8 \%, \mathrm{n}=21$ vs. $1.4 \%$, $\mathrm{n}=5$, respectively, $p<0.001$; Table 1 ).

\section{Modifications of clinical care and treatment strategies}

A modification in the treatment strategy (between the treatments proposed during MTB and those finally received) was reported in $13.1 \%$ ( $n=88$ ) of patients, with no differences between the 2 periods ( $13.3 \%, \mathrm{n}=39$ in 2020 vs. $13 \%, \mathrm{n}=49$ in 2019; $p=0.91$; Fig. 3).

No differences were observed in the treatment distribution: neither for the treatment intent (curative, palliative, or BSC) nor class (interventional, non-interventional, or BSC), as shown in Fig. 4.

The main reasons for the modification of treatment strategy were significantly different in 2020 compared with 2019: COVID19 infection (46.1\% in 2020 and 0\% in 2019), and tumour progression (23.1\% in 2020 and 65.3\% in 2019; Table 1).

\section{Subgroup analysis}

Subgroup with active HCC, first diagnosis (Table 2)

Among the 247 patients with a first diagnosis of HCC ( $n=104$ in 2020 vs. $n=143$ in 2019), the tumour size was significantly larger in 2020 (49 [25-80] mm) compared with 2019 (32 [22-60] mm, $p=0.002$ ) with no significant increase in AFP level in the 2 periods (Table 2).

Subgroup with active, recurrent HCC (Table 3)

This subgroup of 423 patients $(\mathrm{n}=189$ in 2020 vs. $\mathrm{n}=234$ in 2019) presented similar characteristics between the 2 periods. Nevertheless, the rate of patients with a delay of treatment longer than 1 month was significantly higher in 2020 vs. 2019 ( $\mathrm{n}=44,23.3 \%$ vs. $\mathrm{n}=11,4.7 \%$, respectively; $p<0.001$; Table 3 ). When focusing only on the COVID-19 period, we observed

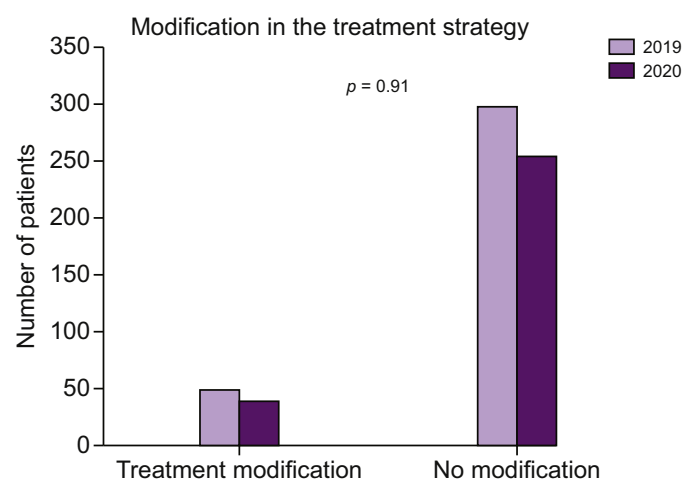

Fig. 3. Modification of treatment strategy. Difference between the treatment proposed to the MTB and the treatment actually received, during the study periods (13.3\%, n = 39 in 2020 vs. 13\%, n = 49 in 2019; Fisher's exact test, $p=$ 0.909). MTB, multidisciplinary tumour board.

differences between the classes of treatment with an MTB-totreatment delay $>1$ month or $<1$ month (Table S2).

Subgroup of patients affected by COVID

Among patients exposed to the COVID-19 pandemic, 7.1\% (21/ 293) had a diagnosis of an active COVID-19 infection. Patients were male in $62 \%$ of cases and $66.2(43.5,73.6)$ years old at diagnosis. This latter was based on PCR and CT scan or CT scan alone in more than two-thirds of patients $(n=8,38.1 \%$ and $n=7$, $33.3 \%$, respectively), followed by PCR alone or typical symptoms of COVID-19 (both $n=3,14.3 \%$ ). Eleven patients (52.4\%) were hospitalised with a median length of stay of $7.00(2.00,28.0)$ days, and 5 patients needed hospitalisation in an intensive care unit (ICU) but 3 were refused ICU admission. Two patients (9.5\%) developed acute respiratory distress syndrome in our group, the rate of complications was similar to patients with other cancer. ${ }^{13}$ Their medical treatment was highly heterogeneous, including antibiotic regimen, hydrochloroquine, and antivirals. Overall, 4 patients died (19.1\%), including the 2 hospitalised in ICU (Table S3), with a median OS of $26.0(3.00,65.0)$ days.
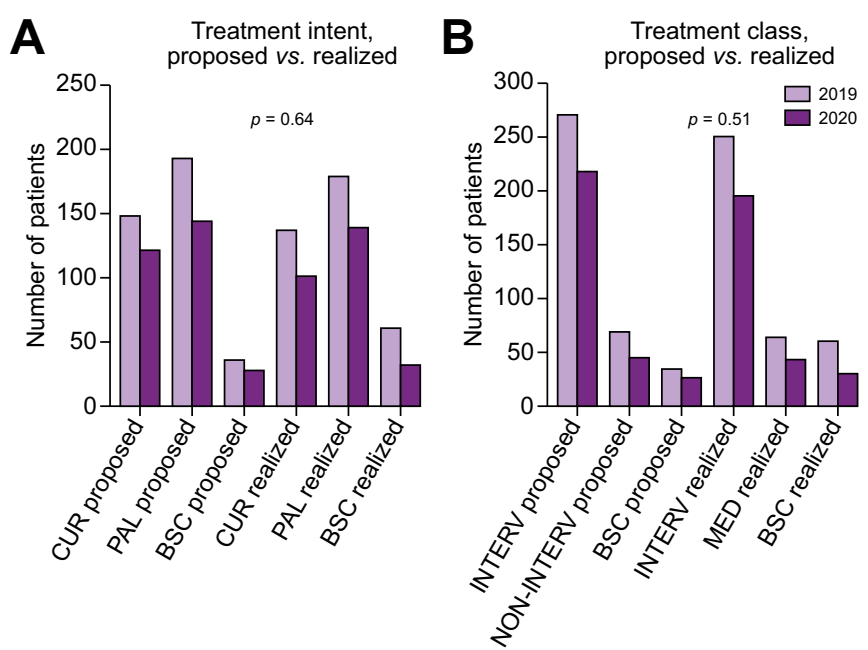

Fig. 4. Modification in treatment intent (A) or class (B), between the 2 time periods. Comparison between years was performed using Fisher's exact test. BSC, best supportive care; CUR, curative; INTERV, interventional treatment; NON-INTERV, non-interventional treatment; PAL, palliative. 
Table 2. Baseline characteristics of 247 patients with first diagnosis of HCC.

\begin{tabular}{|c|c|c|c|}
\hline & $\begin{array}{r}\text { Unexposed COVID-19 } \\
2019(\mathrm{n}=143) \\
\end{array}$ & $\begin{array}{r}\text { Exposed COVID-19 } \\
2020(n=104) \\
\end{array}$ & $\boldsymbol{p}$ value $^{* *}$ \\
\hline Sex - male, n (\%) & $124(86.7)$ & $90(86.5)$ & 1 \\
\hline Age, years & $67(60-74)$ & $69(61-74)$ & 0.548 \\
\hline Cirrhosis, n (\%) & $116(81.1)$ & $81(77.9)$ & 0.631 \\
\hline \multicolumn{4}{|l|}{ Liver disease aetiology, n (\%) } \\
\hline $\mathrm{HCV}$ & $20(14.0)$ & $17(16.3)$ & \\
\hline HBV & $28(19.6)$ & $15(14.5)$ & \\
\hline $\mathrm{HCV}+\mathrm{HBV}$ & $19(13.3)$ & $13(12.5)$ & \\
\hline Alcohol & $28(19.6)$ & $21(20.2)$ & 0.440 \\
\hline NASH & $23(16.1)$ & $25(24.0)$ & \\
\hline Alcohol + NASH & $17(11.9)$ & $10(9.6)$ & \\
\hline Other & $8(5.6)$ & $3(2.9)$ & \\
\hline Tumour burden, $\mathrm{mm}(\mathrm{n}=242)$ & $32(22-60)$ & $49(25-80)$ & 0.022 \\
\hline \multicolumn{4}{|l|}{ BCLC classification } \\
\hline BCLC 0 & $11(7.7)$ & $7(6.7)$ & \\
\hline BCLC A & $60(42.0)$ & $46(44.2)$ & \\
\hline BCLC B & $27(18.9)$ & $21(20.2)$ & 0.780 \\
\hline BCLC C & $27(18.9)$ & $18(17.3)$ & \\
\hline BCLC D & $18(12.6)$ & $12(11.5)$ & \\
\hline Tumour thrombosis & $26(18.2)$ & $20(19.2)$ & 0.869 \\
\hline \multicolumn{4}{|l|}{ Alpha-fetoprotein, ng/ml $(\mathrm{n}=241)$} \\
\hline$<10$ & $66(47.1)$ & $38(37.6)$ & 0.149 \\
\hline$>10$ & $74(52.9)$ & $63(62.4)$ & \\
\hline \multicolumn{4}{|l|}{ Inclusion criteria, n (\%) } \\
\hline LS and IR performed & $30(21.0)$ & $21(20.2)$ & 1 \\
\hline MTB discussion & $113(79.0)$ & $83(79.8)$ & \\
\hline \multicolumn{4}{|l|}{ Circumstance of diagnosis, $\mathrm{n}(\%)$} \\
\hline By chance & $25(17.4)$ & $17(16.3)$ & \\
\hline Screening & $72(50.3)$ & $45(43.6)$ & 0.401 \\
\hline Symptomatic & $46(32.2)$ & $42(40.8)$ & \\
\hline \multicolumn{4}{|l|}{ Diagnostic modality, n (\%) } \\
\hline Imaging & $106(74.1)$ & $72(69.2)$ & 0.473 \\
\hline Histology & 37 (25.9) & $32(30.8)$ & \\
\hline \multicolumn{4}{|l|}{ Imaging technique, $n(\%)(n=220)$} \\
\hline $\mathrm{CT}$ & $39(31.7)$ & $30(30.9)$ & \\
\hline MRI & $44(35.8)$ & $41(42.3)$ & 0.551 \\
\hline Both & $40(32.5)$ & $26(26.8)$ & \\
\hline \multicolumn{4}{|l|}{ Proposed treatment } \\
\hline Curative & $68(47.6)$ & $50(48.1)$ & \\
\hline Palliative & $43(37.1)$ & $40(38.5)$ & 0.576 \\
\hline BSC & $22(15.4)$ & $14(13.5)$ & \\
\hline \multicolumn{4}{|l|}{ Performed treatment } \\
\hline Curative & $63(44.1)$ & $44(42.3)$ & \\
\hline Palliative & $48(33.6)$ & $40(38.5)$ & 0.530 \\
\hline BSC & $31(21.7)$ & $17(16.3)$ & \\
\hline None performed & $1(0.7)$ & $3(2.9)$ & \\
\hline Therapeutic protocol inclusion & $10(7.0)$ & $2(1.9)$ & 0.078 \\
\hline Treatment change & $22(15.4)$ & $12(11.5)$ & 0.456 \\
\hline \multicolumn{4}{|l|}{ Cause of treatment change } \\
\hline Tumour progression & $12(54.5)$ & $2(16.7)$ & \\
\hline Failed or contra-indicated treatment & $9(40.9)$ & $2(16.7)$ & $<0.001$ \\
\hline Patient's choice & $1(4.5)$ & $1(8.3)$ & \\
\hline Related to COVID-19 & $0(0)$ & $7(58.3)$ & \\
\hline \multicolumn{4}{|l|}{ MTB-to-treatment interval, days } \\
\hline Curative* & $43(24-61)$ & $31(9-47)$ & 0.034 \\
\hline Palliative & $30(16-43)$ & $22(13-31)$ & 0.109 \\
\hline BSC & $0(0-1)$ & $0(0-2)$ & 0.962 \\
\hline MTB-to-treatment interval $>1$ month & $25(17.5)$ & $19(18.3)$ & 0.868 \\
\hline \multicolumn{4}{|l|}{ Outpatient consultation } \\
\hline Cancelled & $5(3.5)$ & $16(15.4)$ & \\
\hline Standard & $138(96.5)$ & $68(65.4)$ & $<0.001$ \\
\hline Teleconsultation & $0(0)$ & $20(19.2)$ & \\
\hline
\end{tabular}

BSC, best supportive care; HCC, hepatocellular carcinoma; IR, interventional radiology; LS, liver surgery; MTB, multidisciplinary tumour board; NASH, non-alcoholic steatohepatitis.

${ }^{* *}$ Categorical variables were compared using Fisher's exact test and continuous variables using Mann Whitney non parametric test.

* LT and the related work up were excluded, only 4 cases of TH. 
Table 3. Baseline characteristics of 423 patients with recurrent active HCC.

\begin{tabular}{|c|c|c|c|}
\hline & $\begin{array}{r}\text { Unexposed COVID-19 } \\
2019(\mathrm{n}=234) \\
\end{array}$ & $\begin{array}{r}\text { Exposed COVID-19 } \\
2020(n=189) \\
\end{array}$ & $p$ value $^{* *}$ \\
\hline Age, years & $67(60-74)$ & $66(60-73)$ & 0.549 \\
\hline \multicolumn{4}{|l|}{ Liver disease aetiology, n (\%) } \\
\hline $\mathrm{HCV}$ & $43(18.4)$ & $34(18.0)$ & \\
\hline HBV & $31(13.2)$ & $23(12.2)$ & \\
\hline $\mathrm{HCV}+\mathrm{HBV}$ & $22(9.4)$ & $31(16.4)$ & 0.542 \\
\hline Alcohol + NASH & $40(17.1)$ & $28(14.8)$ & \\
\hline Other & $17(7.3)$ & $12(6.3)$ & \\
\hline Tumour burden, $\mathrm{mm}(\mathrm{n}=398)$ & $27(16-50)$ & $25(16-45)$ & 0.545 \\
\hline \multicolumn{4}{|l|}{ BCLC classification $(\mathrm{n}=404)$} \\
\hline BCLC 0 & $24(11.0)$ & $21(11.4)$ & \\
\hline BCLC A & $58(26.5)$ & 59 (31.9) & \\
\hline$<10$ & $126(55.5)$ & $101(54.6)$ & 0.921 \\
\hline$>10$ & $101(44.5)$ & $84(45.4)$ & \\
\hline \multicolumn{4}{|l|}{ Inclusion criteria, $\mathrm{n}(\%)$} \\
\hline LS and IR performed & $43(18.4)$ & $51(27.0)$ & 0.045 \\
\hline MTB discussion & $191(81.6)$ & $138(73.0)$ & \\
\hline \multicolumn{4}{|l|}{ Diagnostic modality, n (\%) } \\
\hline Imaging & $182(77.8)$ & $157(83.1)$ & 0.643 \\
\hline Histology & $52(22.2)$ & $32(16.9)$ & \\
\hline \multicolumn{4}{|l|}{ Imaging technique, $n(\%)(n=393)$} \\
\hline CT & 79 (36.9) & $60(33.5)$ & \\
\hline MRI & $74(34.6)$ & $58(32.4)$ & 0.499 \\
\hline Both & $61(28.5)$ & $61(34.1)$ & \\
\hline \multicolumn{4}{|l|}{ Proposed treatment } \\
\hline Treatment change & $27(11.5)$ & $27(14.3)$ & 0.464 \\
\hline \multicolumn{4}{|l|}{ Cause of treatment change $(n=53)$} \\
\hline Disease progression & $20(74.1)$ & $6(22.2)$ & \\
\hline Failed or contra-indicated treatment & $5(18.5)$ & $2(7.4)$ & $<0.001$ \\
\hline Patient's choice & $2(7.4)$ & $6(22.2)$ & \\
\hline Related COVID-19 & $0(0)$ & $13(48.1)$ & \\
\hline \multicolumn{4}{|l|}{ MTB-to-treatment interval, day } \\
\hline Curative* & $31(22-40)$ & $31(18-60)$ & 0.445 \\
\hline Palliative & $22(8-36)$ & $27(11-43)$ & 0.116 \\
\hline BSC & $1(0-17)$ & $4(0-23)$ & 0.871 \\
\hline MTB-to-treatment interval $>1$ month & $11(4.7)$ & $44(23.3)$ & $<0.001$ \\
\hline \multicolumn{4}{|l|}{ Outpatient consultation } \\
\hline Cancelled & $4(1.7)$ & $6(3.2)$ & \\
\hline Standard & $226(96.6)$ & $97(51.3)$ & $<0.001$ \\
\hline Teleconsultation & $4(1.7)$ & $86(45.5)$ & \\
\hline
\end{tabular}

BSC, best supportive care; HCC, hepatocellular carcinoma; IR, interventional radiology; LS, liver surgery; MTB, multidisciplinary tumour board; NASH, non-alcoholic steatohepatitis.

${ }^{* *}$ Categorical variables were compared using Fisher's exact test and continuous variables using Mann Whitney non parametric test

* LT and the related work up were excluded.

Multivariate logistic regression for predictors of intention to treat strategy change (Table 4)

In univariate analysis, the diagnosis status (first diagnosis or follow up), the type of treatment proposed and the period (2019 vs. 2020) were associated with a significant delay or change in strategy of treatment. On multivariate analysis, only the period in 2020 was independently associated with delay or change in strategy (aOR = 9.661 [95\% CI: 2.85-32.72], $p<0.001$; Table 4).

When focusing on variables associated with a treatment delay longer than 1 month, the period and the type of 
Table 4. Uni- and multivariate logistic regression analysis for changed/delayed treatment and for delay of treatment $\geq 1$ month.

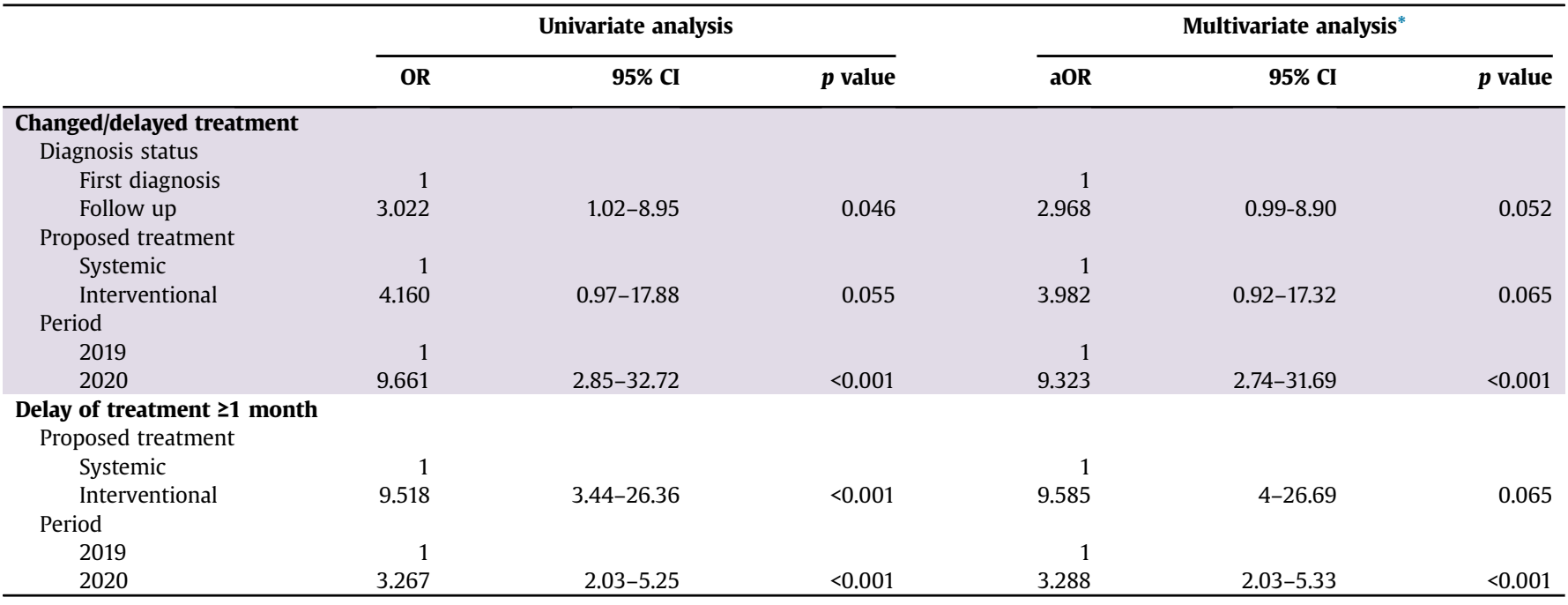

${ }^{*}$ Hosmer Lemeshow test: changed/delayed treatment $=0.967$; aOR adjusted for type of management and type of treatment; delay of treatment $\geq 1$ month $=0.956$. aOR, adjusted odds ratio.

treatment proposed were found to be significant using univariate analysis. After adjusting, only the period in 2020 was independently associated $(\mathrm{aOR}=9.323,95 \% \mathrm{Cl}: 2.74-31.69, p$ $<0.001$; Table 4).

\section{Discussion}

The COVID-19 pandemic has suddenly shattered the processes of healthcare in general, and for patients affected by cancer in particular. In France, similarly to other Western countries, planned clinical activities were reduced and postponed to minimise the risk of viral transmission but also to allow the re-assignment of health professionals to support COVID-19 units. Recommendations ${ }^{14}$ by scientific societies try to deal with the current situation, but the real impact of the COVID-19 pandemic in HCC management is still unknown. A recent multicentre Italian study reported the outcomes of COVID-19 infection in patients with cirrhosis. ${ }^{15}$ In contrast, our study focused on HCC and the treatment received in this period.

In this study, involving 6 referral academic centres within the metropolitan area of Paris, highly affected by the pandemic, we observed a significant decrease over the weeks in the rate of patients with HCC referred for first diagnosis or treatment (proposed or performed). The interpretation of these findings may rely on a complex set of correlated reasons, including an increased delay of consultation of patients for symptoms to their general practitioner, a decreased referral by other professionals because of fear of COVID-19 infection, and reduced access to diagnostic tools, operating theatres, and ICU. The lower number of new patients with HCC diagnosed during the COVID-19 period could be also explained by the travel limitation which prevented patients from other areas reaching medical centres in Paris.

Hence, the modification in the treatment strategy (curative $v s$. palliative, and interventional vs. non-interventional) were not different in 2020 from 2019. A panel of experts published recommendations about treatment modification and downgrading $^{16}$ for patients with HCC in the COVID-19 pandemic. However, the period of the current study was at the beginning of the lockdown, before these recommendations were published.
The rate of patients with a treatment delay longer than 1 month was significantly higher in 2020 compared with 2019, and this observation is supported by multivariate analysis, in which the period 2020 was found to be a strong independent predictor of treatment delay or cancellation. This finding could be explained by a decreased access to operating theatres, interventional radiology facilities, post-operative ICUs, and ventilators, forcing physicians to delay patient treatment. However, we found a shorter interval between presentation to the multidisciplinary tumour board and treatment in 2020 compared with 2019. This difference could be explained by the high numbers of treatments delayed in 2020 after the period considered. Consequently, the few patients treated during the COVID-19 period in 2020 has a shorter delay to treatment compared with 2019. Most of the patients with a treatment delayed for more than 1 month have a recurrent HCC. These data suggested that physicians tended to treat patients with a new HCC more quickly during the COVID-19 period. We could hypothesise that patients with new HCC tend to be treated quickly because of the lack of knowledge of tumour biology and the pressure of patients who received a recent diagnosis of cancer. In patients with HCC recurrence, the physician tends to be familiar with the tumour biology and could adapt the delaying of treatment accordingly.

Not surprisingly, the significant shift towards the rates of teleconsultation in 2020 vs. 2019 is related to social distancing measures as well as patient fear, as a result of the pandemic. The impact of teleconsultation on the patient-physician relationship, as well as on the patient's understanding of HCC diagnosis and treatment should be further explored.

Finally, $7.1 \%$ of patients exposed to the pandemic were affected by COVID-19, and almost half of them required hospitalisation. With 4 deaths (19\%) the crude fatality rate in this study population is significantly higher than that reported in China, ${ }^{4,5}$ Italy, ${ }^{6}$ or the $\mathrm{UK},{ }^{17}$ but is consistent with reported French government data. ${ }^{10}$ The reduced number of infected patients and the competitive risk of death of cancer and comorbidities prevent any reliable conclusion. The mortality rate (19.2\%) was lower than that observed at 30 days (34\%) in patients with cirrhosis (mostly without HCC) in an Italian multicentre 
study. ${ }^{15}$ Of note, fewer patients required hospitalisation in our series (52.4\%) compared with the Italian cohort (96\%) suggesting a different severity profile at baseline.

We have to emphasise that the COVID-19 diagnosis was performed by clinicians and was not a systematic prospective assessment of patient symptoms with a pre-defined PCR or CT scan-based diagnostic algorithm.

The main limit of this study is represented by the uncertain delay of treatment reported in 2020 (at the time of the study, some $7 \%$ of patients had not received the treatment planned) preventing calculation of a reliable delay that could be compared with 2019. In this study, we could not assess the impact on patient survival of treatment delay and of the reduced rates of MTB presentations. Moreover, the observations reported in this study could not be generalised to areas with a low incidence of COVID-19 infection.

To better understand the impact of COVID-19 on HCC management, we will need to gather the reasons for the reduction in
MTB presentations and the delay in treatments. These data would be helpful to improve access to clinical care for patients affected by cancer, and could be generalised to other types of cancers, in case of future pandemics.

This study is a very early snapshot (6 weeks) of the French lockdown (10 weeks); it offers the first report of a homogeneous population affected by HCC within a network of high-volume academic French centres in a COVID-19 pandemic area.

Based on the available data, the pandemic seems to impact the management of patients affected by HCC in one-quarter of the population, owing to a delay in treatment realisation but not with a modification in treatment strategy.

The mid-term follow up of this cohort will inform about the impact of the pandemic on the long-term HCC management, waitlist dropout, and mortality.

\begin{abstract}
Abbreviations
aOR, adjusted OR; BCLC, Barcelona Clinic Liver Cancer; EASL, European Association for the Study of Liver; HCC, hepatocellular carcinoma; ICU, intensive care unit; IQR, inter-quartile range; IR, interventional radiology; ITT, intention to treat; LR, liver resection; LT, liver transplantation; MELD, model for end-stage liver disease; MTB, multidisciplinary tumour board; $\mathrm{NASH}$, non-alcoholic steatohepatitis; OR, odds ratio; SIRT, selective internal radiation therapy; TACE, transarterial chemoembolisation.
\end{abstract}

\section{Conflict of interest}

JCN received a research grant from Bayer for INSERM UMR1138. The other authors declare no conflicts of interest that pertain to this study.

Please refer to the accompanying ICMJE disclosure forms for further details.

\section{Authors' contributions}

Conceived and organised the study, planned the data management, organised data collection for the steering centre and contributed to the statistical analyses, wrote the manuscript and figures, obtained the IRB approval, gave their final approval of the manuscript: GA, RB.

Organised data collection for each participating centre, significantly contributed to the manuscript and gave the final approval before submission: JCN, MA, ML, CH, HR, MB.

Planned and realised the statistical analyses, significantly contributed to the manuscript and gave her final approval before submission: FRT.

Revised the manuscript and gave their final approval before submission: all remaining authors.

\section{Data availability}

Research data are not available for sharing given their confidential nature.

\section{Supplementary data}

Supplementary data associated with this article can be found, in the online version, at https://doi.org/10.1016/j.jhepr.2020.100199.

\section{References}

[1] Coronavirus disease 2019. Available at: https://www.who.int/ emergencies/diseases/novel-coronavirus-2019?gclid=Cj0KCQjwzZj2BRDV ARIsABs319KnvaU-065-0zz6tR7Qr-ZRzsyzY5TEU3mO-mdhSLe9j9Kx72bTg cUaAg4FEALw_wcB. [Accessed 21 May 2020].

[2] Bray F, Ferlay J, Soerjomataram I, Siegel RL, Torre LA, Jemal A. Global cancer statistics 2018: GLOBOCAN estimates of incidence and mortality worldwide for 36 cancers in 185 countries. CA Cancer J Clin 2018;68:394-424.

[3] Gosain R, Abdou Y, Singh A, Rana N, Puzanov I, Ernstoff MS. COVID-19 and cancer: a comprehensive review. Curr Oncol Rep 2020;22:53.
[4] Wu Z, McGoogan JM. Characteristics of and important lessons from the coronavirus disease 2019 (COVID-19) outbreak in China: summary of a report of 72314 cases from the Chinese Center for Disease Control and Prevention. JAMA 2020;323:1239-1242.

[5] Liang W, Guan W, Chen R, Wang W, Li J, Xu K, et al. Cancer patients in SARS-CoV-2 infection: a nationwide analysis in China. Lancet Oncol 2020;21:335-337.

[6] Onder G, Rezza G, Brusaferro S. Case-fatality rate and characteristics of patients dying in relation to COVID-19 in Italy. JAMA 2020;323:17751776.

[7] You B, Ravaud A, Canivet A, Ganem G, Giraud P, Guimbaud R, et al. The official French guidelines to protect patients with cancer against SARSCoV-2 infection. Lancet Oncol 2020;21:619-621.

[8] Boettler T, Newsome PN, Mondelli MU, Maticic M, Cordero E, Cornberg M, et al. Care of patients with liver disease during the COVID-19 pandemic: EASL-ESCMID position paper. JHEP Rep 2020;2:100113.

[9] Ganne-Carrié N, Fontaine H, Dumortier J, Boursier J, Bureau C, Leroy V, et al. Suggestions for the care of patients with liver disease during the Coronavirus 2019 pandemic. Clin Res Hepatol Gastroenterol 2020;44:275-281.

[10] Info coronavirus covid 19 - carte et donnees covid 19 en france, Gouvernement.fr. Available at: https://www.gouvernement.fr/infocoronavirus/carte-et-donnees. [Accessed 21 May 2020].

[11] von Elm E, Altman DG, Egger M, Pocock SJ, Gøtzsche PC, Vandenbroucke JP, et al. The Strengthening the Reporting of Observational Studies in Epidemiology (STROBE) statement: guidelines for reporting observational studies. J Clin Epidemiol 2008;61: 344-349.

[12] Galle PR, Forner A, Llovet JM, Mazzaferro V, Piscaglia F, Raoul JL, et al. EASL clinical practice guidelines: management of hepatocellular carcinoma. J Hepatol 2018;69:182-236.

[13] Kuderer NM, Choueiri TK, Shah DP, Shyr Y, Rubinstein SM, Rivera DR, et al. Clinical impact of COVID-19 on patients with cancer (CCC19): a cohort study. Lancet 2020;395(10241):1907-1918.

[14] Di Fiore F, Bouché O, Lepage C, Sefrioui D, Gangloff A, Schwarz L, et al. COVID-19 epidemic: proposed alternatives in the management of digestive cancers: a French intergroup clinical point of view (SNFGE, FFCD, GERCOR, UNICANCER, SFCD, SFED, SFRO, SFR). Dig Liver Dis 2020;52:597-603.

[15] Iavarone M, D’Ambrosio R, Soria A, Triolo M, Pugliese N, Del Poggio P, et al. High rates of 30-day mortality in patients with cirrhosis and COVID-19. J Hepatol 2020;73:1063-1071.

[16] Barry A, Apisarnthanarax S, O'Kane GM, Sapisochin G, Beecroft R, Salem R, et al. Management of primary hepatic malignancies during the COVID-19 pandemic: recommendations for risk mitigation from a multidisciplinary perspective. Lancet Gastroenterol Hepatol 2020;5: 765-775.

[17] Undela K, Gudi SK. Assumptions for disparities in case-fatality rates of coronavirus disease (COVID-19) across the globe. Eur Rev Med Pharmacol Sci 2020;24:5180-5182. 